\title{
NATIONAL LABORATORY SURVEILLANCE OF INFLUENZA IN MACEDONIA DURING FOUR SEASONS
}

\author{
Golubinka Bosevska1', Vladimir Mikik', Irena Topuzovska Kondova², Nikola Panovski³; Collaborators \\ ${ }^{1}$ Institute of Public Health, Skopje, Republic of Macedonia \\ 2University Clinic for Infectious Diseases, Skopje, Republic of Macedonia \\ ${ }^{3}$ Institute of Microbiology and Parasitology, Medical Faculty, Saints Cyril and Methodius University, Skopje, Republic of Macedonia
}

\section{SUMMARY}

Objective: The aim of the study is to comprehend results of the influenza lab surveillance system in the Republic of Macedonia after the 2009 pandemic and to determine the main characteristics of four consecutive epidemic seasons (from 2010/2011 until 2013/2014).

Methods: As part of the universal surveillance system, nasal and throat specimens were collected from patients. After extraction of RNA, the CDC real-time RT-PCR assays for the detection of influenza types and subtypes were performed.

Results: Out of 920 tested samples, 406 (44.1\%) laboratory confirmed cases of influenza were found. Influenza activity begins as early as December and continues until the end of April with peaks in January or February with predominant influenza A and A/H1N1pdm. Influenza A viruses start their activity at week 49 to 52 and subside at week 17. Usually two peaks appear, the first one between week 2 and 4 and the second one between week 6 and 9. Subtype A/H1N1pdm was dominant among influenza A types in the 2010/2011 and 2012/2013 seasons. A/H3N2 was the only circulating influenza virus in the 2011/2012 season. Influenza B season is shorter and has only one peak, between weeks $2-5$. Usually the influenza B viruses emerge in later stages than influenza A viruses, except for the first post-pandemic season.

Conclusion: Results revealed that post-pandemic influenza seasons in Macedonia were rather different. Although the influenza season pattern is similar to patterns in some countries of the WHO European region, some unique characteristics were observed.

Key words: influenza, laboratory surveillance, post-pandemic, influenza subtypes

Address for correspondence: G. Bosevska, Vasil Gjorgov br 20/49, 1000 Skopje, Republic of Macedonia. E-mail: golubinka@yahoo.com; golubinkavm@gmail.com

https://doi.org/10.21101/cejph.a4272

\section{INTRODUCTION}

Influenza prevention is one of the public health priorities all over the world. Since 2003, in collaboration with many partners, the WHO has developed the Global Agenda on Influenza Surveillance and Control defining 17 priority activities that are critical to reducing the morbidity and mortality from annual influenza epidemics and preparing for the next pandemic. The activities are organized around four main objectives: strengthening surveillance, improving knowledge of the disease burden, increasing vaccine usage, and accelerating pandemic preparedness. One of the main actions is the rapid identification of all influenza virus subtypes (1). International Health Regulation 2005 requires all WHO members "to enhance the capacities for surveillance and response", including influenza surveillance (2).

The pandemic influenza A/H1N1pdm in 2009 and the announcement of pandemic phase 6 by the WHO clearly demonstrated the need for better understanding and continuous surveillance of influenza, especially the importance of enhancing the laboratory and epidemiological surveillance (3). Influenza seasons can be unpredictable regarding the beginning, severity, types and subtypes of circulating virus, thus matching circulating viruses and viruses in the vaccine can be challenging. Efficient surveillance systems that constitute both epidemiological surveillance and virological surveillance enable effective and rapid detection and control of threats related to influenza.

The aim of this paper is to comprehend results of the laboratory surveillance system for influenza in Macedonia after the 2009 pandemic and to determine the main characteristics of four annual influenza epidemics.

\section{MATERIALS AND METHODS}

In the Republic of Macedonia, influenza is a mandatory notifiable disease. According to the Law on the Protection of Population from Communicable Diseases (4) and based on the Annual Preventive Programme, there is obligation to conduct routine epidemiological surveillance of influenza. There are two modalities of the reporting system depending on the time of season. The first is the 'out of season' reporting system from week 21 until week 39 of the current year, when influenza-like illness (ILI) cases are reported on individual reporting forms. The second is the seasonal reporting system from week 40 until week 20 of the following 
year, when ILI cases are reported on aggregated reporting forms divided by age groups. It is mandatory to provide notification of laboratory confirmed cases of influenza on individual reporting forms according to the Rulebook for Reporting Communicable Diseases (5). Reports weekly, monthly and annually prepared by the Institute of Public Health (IPH) are distributed to the Ministry of Health, regional and sub-regional Public Health Centres, hospitals, general practitioners (GPs), health insurance institute, and the WHO Regional Office for Europe.

For the purpose of virological surveillance, epidemiologists from 21 sub-regional units and 10 regional Public Health Centres were assigned to collect samples of suspected influenza cases in their region and send them to the national influenza laboratory of IPH for virology and molecular diagnostics. Samples for laboratory testing were collected from the University Clinic for Infectious Diseases where patients with severe influenza were hospitalized. The selection of patients followed the ECDC ILI case definition and acute respiratory illness (ARI) case definition (6).

For the purpose of laboratory surveillance, nasal and throat specimens were collected, placed in viral transport media, and stored at $4{ }^{\circ} \mathrm{C}$ until transported to the laboratory. Upon receipt at the lab, RNA extractions were performed using the QIAamp viral RNA kit (Qiagen, Valencia, CA, USA). The RNA was tested for the presence of all influenza virus types and specific subtypes using the CDC real-time RT-PCR assay directed at the matrix gene sequences of influenza $A$ and $B$, and hemagglutinin gene sequences for influenza A subtypes - H1 pandemic, H3 seasonal, and H1 seasonal. The laboratory testing of samples was performed in accordance with the described laboratory diagnostic protocol (7).

In total, 920 samples were received at the laboratory for influenza testing during four post-pandemic seasons of influenza: 2010/2011, 2011/2012, 2012/2013 and 2013/2014 (Table 1).

The number of samples sent for laboratory testing differed in every season ranging from 160 samples in 2011/2012 season to 349 samples in the first season after the pandemic in 2010/2011.

\section{RESULTS}

A total of 406 laboratory-confirmed cases of influenza were reported from the beginning of influenza season 2010/2011, week 40 of 2010 until the end of the 2013/2014 season, week 20 of 2014.

The total number of laboratory-confirmed cases of seasonal influenza (all types) per week from 2010-2014 is shown in Figure 1. The lowest number of positive samples was 61 , detected in the 2011/2012 season, whereas the highest was 124 positive samples in the 2010/2011 season, which correlates with the volume of suspected cases sent in for laboratory testing each season. In the first two post-pandemic seasons the proportion of positive samples was lower $(\geq 35 \%)$ in comparison with the proportion of positive samples in two following seasons (more than 52\%).

The earliest start among all four seasons was 2010/2011 with detection of the first positive samples in week 46 (middle of November), slow growth from week 52 (second part of December), reaching the peak in week 4 of the following year. Actually, in the first three post-pandemic seasons the peak was reached in week 4 of the respective year. Only in the 2013/2014 season was the peak reached later in week 7 . The majority of samples were detected in the period from week 6 (second week of February) to week 9 (second week of March) in the first two seasons, whereas

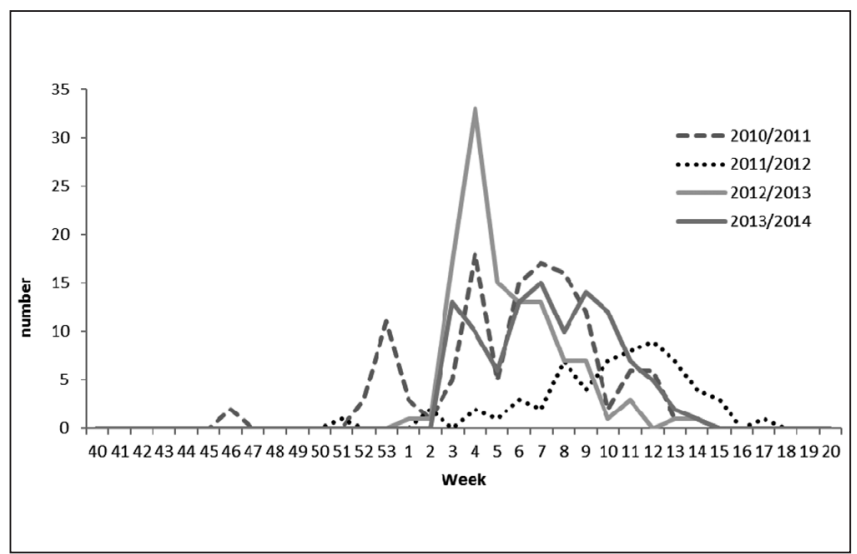

Fig. 1. Total number of laboratory-confirmed cases of seasonal influenza (all types) per week, 2010-2014, n=406.

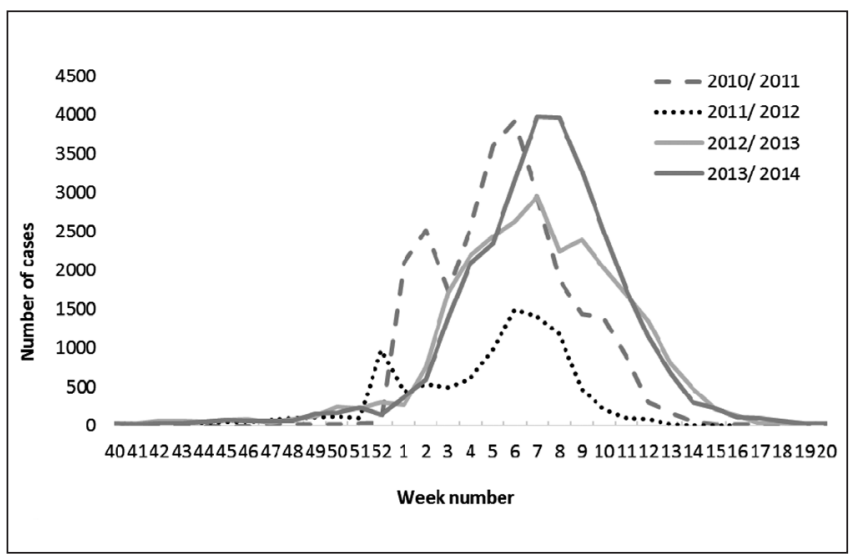

Fig. 2. Weekly number of ILI cases reported through surveillance system for communicable diseases in Macedonia, 2010-2014, $n=90,459$.

Table 1. Number of tested and number of positive influenza samples during four post pandemic seasons

\begin{tabular}{|l|c|c|c|c|c|c|}
\hline Season & $\begin{array}{c}\text { Number of tested } \\
\text { samples }\end{array}$ & $\begin{array}{c}\text { Number of positive } \\
\text { samples (\%) }\end{array}$ & Influenza A (\%) & $\begin{array}{c}\text { Influenza } \\
\text { A/H1N1pdm (\%) }\end{array}$ & $\begin{array}{c}\text { Influenza A/H3N2 } \\
(\%)\end{array}$ & Influenza B (\%) \\
\hline $2010 / 2011$ & 349 & $124(35.5)$ & $85(68.5)$ & $71(83.5)$ & $5(5.9 \%)$ & $39(31.5)$ \\
\hline $2011 / 2012$ & 160 & $61(38.1)$ & $61(100.0)$ & 0 & $52(85.4)$ & 0 \\
\hline $2012 / 2013$ & 205 & $113(55.1)$ & $96(84.9)$ & $70(72.9)$ & $14(14.6)$ & $17(15.1)$ \\
\hline $2013 / 2014$ & 206 & $108(52.4)$ & $96(88.9)$ & $12(12.5)$ & $34(35.4)$ & $12(11.1)$ \\
\hline Total & 920 & $406(44.1)$ & $338(83.2)$ & $153(45.2)$ & $105(31.1)$ & $68(16.7)$ \\
\hline
\end{tabular}


the majority of samples in two following seasons were detected from week 3 (second part of January) until week 7 (end of the first part of February).

A comparison between laboratory surveillance with epidemiological data from the communicable diseases surveillance system in the country showed that first peaks in laboratory surveillance data correspond with the start of the season (Figure 2). According to virological data for the period 2010-2014 in Macedonia, influenza A viruses start their activity at the end of December (weeks 49 to 52), and usually have two peaks - one at the begining of January (weeks 2 to 4) and the second one in February (weeks 6 to 9). The influenza season ends around week 16 to 17 (Figures 3, 4 and 5).

According to the data from virological surveillance for the period 2010-2014, the influenza B season is shorter than the one of influenza A and usually has only one peak during January (weeks 2 to 5) with sporadic cases until April (weeks 14 to 15) (Figure 6).

The domination of influenza type and subtype is different not only among seasons, but also during one season. In the first post-pandemic season $(2010 / 2011)$, out of 124 positive influenza samples, 85 (68.55\%) were positive for influenza A and 39 (31.45\%) were positive for influenza B (Figure 7). The influenza season started with detection of influenza B in week 52 (last week of December), with two clear peaks, first at week 53 and the second in week 4. Influenza A activity started one month later

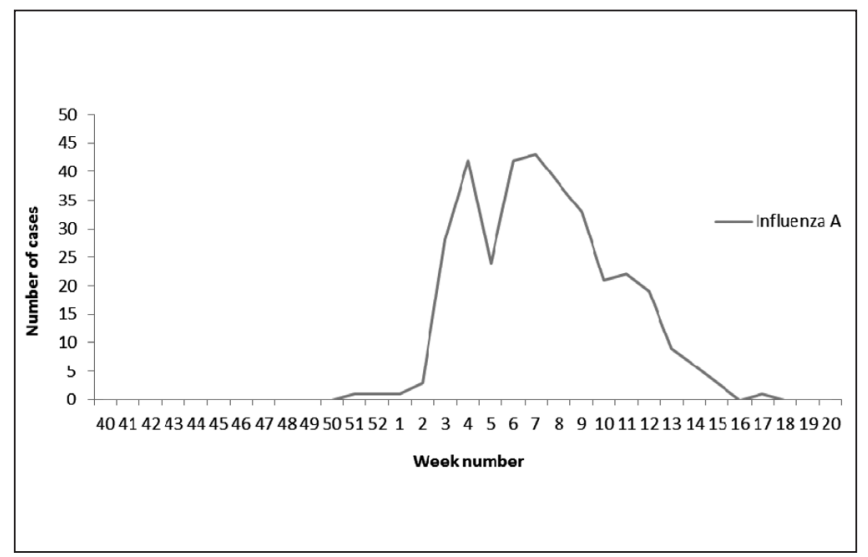

Fig. 3. Total number of laboratory-confirmed influenza $A$ cases, 2010-2014, $n=337$.

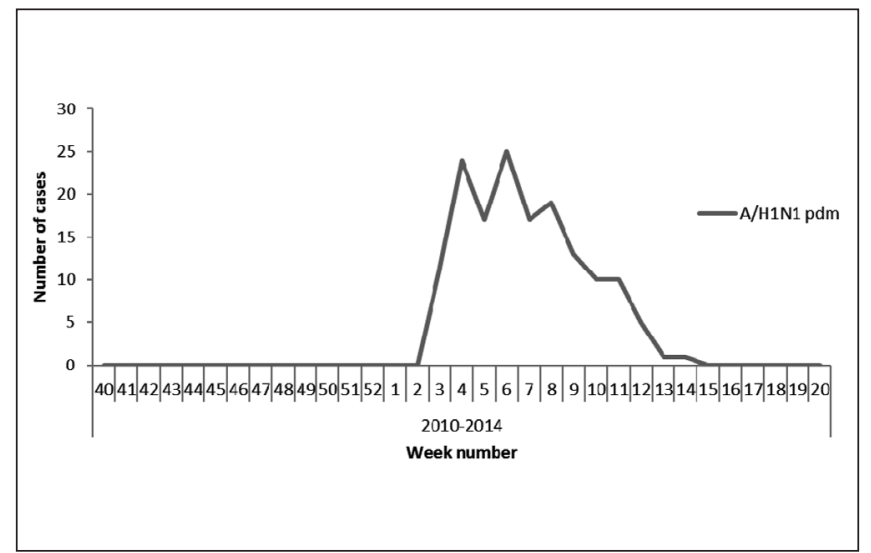

Fig. 4. Total number of laboratory-confirmed cases of influenza A/H1N1, 2010-2014, $n=153$.

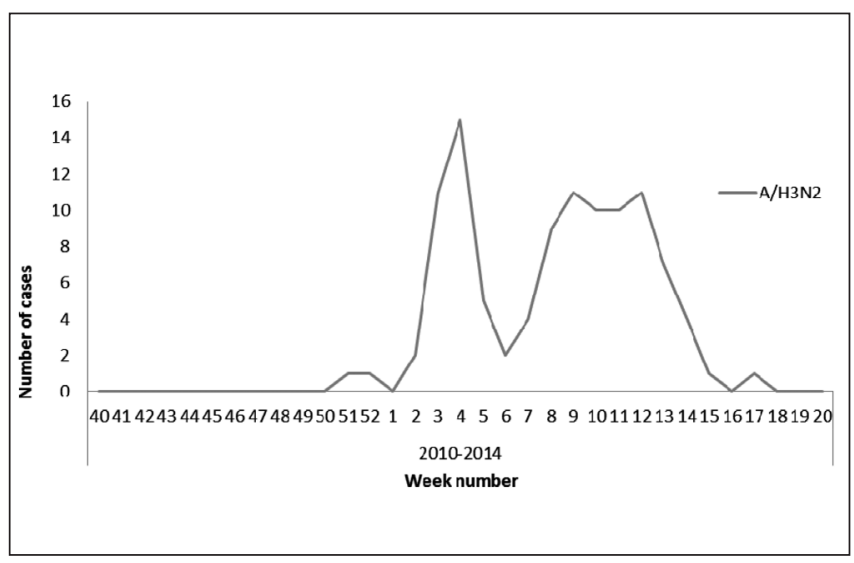

Fig. 5. Total number of laboratory-confirmed cases of influenza A/H3N2, 2010-2014, $n=105$.

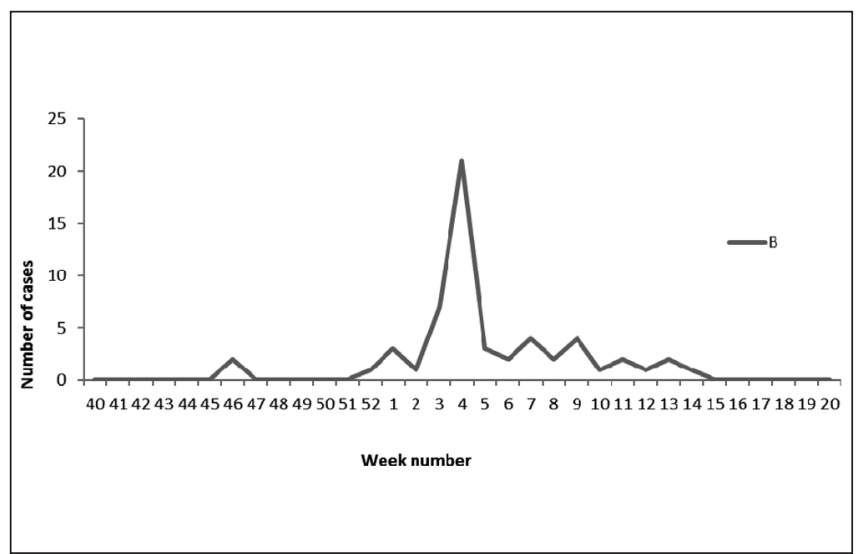

Fig. 6. Total number of laboratory-confirmed cases of influenza $B, 2010-2014, n=64$.

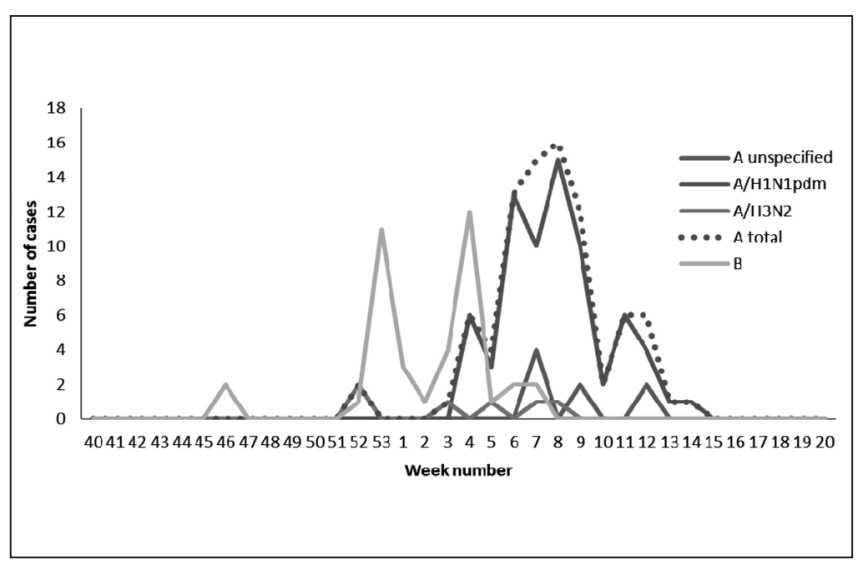

Fig. 7. Number of laboratory-confirmed cases of seasonal influenza (all types) per week, 2010/2011, $n=124$.

than influenza B, actually at week 4 (last week of January), with a peak at week 8 (last week of February). Subtype A/H1N1pdm was dominant among influenza A types with 71 positive samples $(83.53 \%)$. The influenza B activity subsided at week 7 (middle of February), while influenza A activity continued until week 14 (first week of April).

During the 2011/2012 season, out of 160 influenza samples tested, 61 (38.1\%) were positive for influenza A (Figure 8). In- 


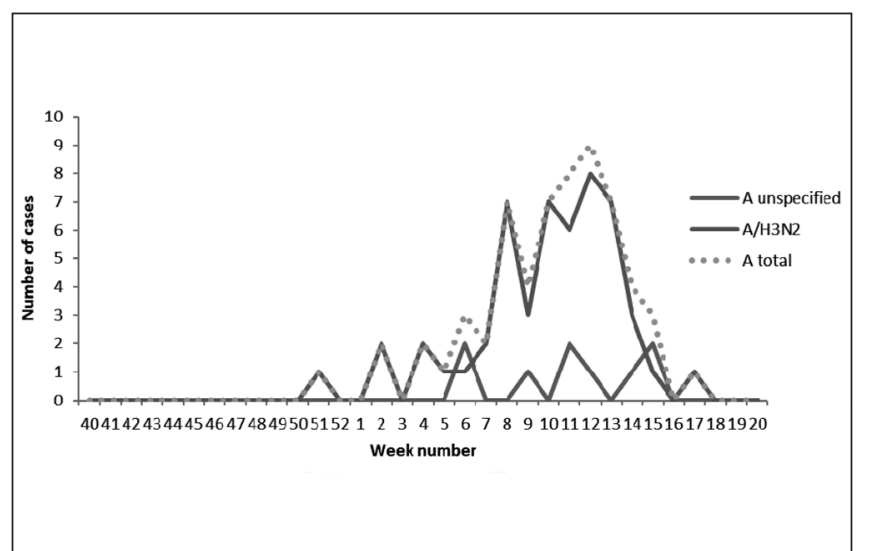

Fig. 8. Number of laboratory-confirmed cases of seasonal influenza (all types) per week, 2011/2012, $n=61$.

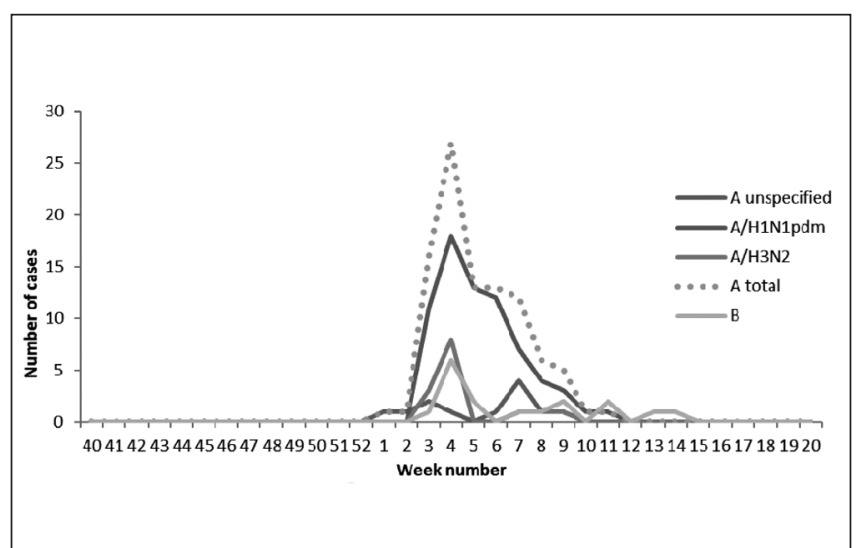

Fig. 9. Number of laboratory-confirmed cases of seasonal influenza (all types) per week, 2012/2013, $n=113$.

fluenza A/H3 started at week 51 (second part of December), with high activity between weeks 8 and 13 (end of February until end of March). The season finished at week 17 (last week of April).

Out of 205 samples tested in 2012/2013 season, 113 samples were positive for influenza. Of them $96(84.96 \%)$ were influenza A samples, whereas 17 (15.04\%) were influenza B samples (Figure 9). A proportion of 70 (72.92\%) influenza A samples were H1N1pdm, while 14 (14.6\%) were influenza A/H3N2. The influenza season started at week 1 (January) as influenza A. The peak of occurrence of all types and subtypes of influenza virus was reached at week 4 (second part of January) with domination of influenza $\mathrm{A} / \mathrm{H} 1 \mathrm{~N} 1 \mathrm{pdm}$. The last detection of influenza A virus

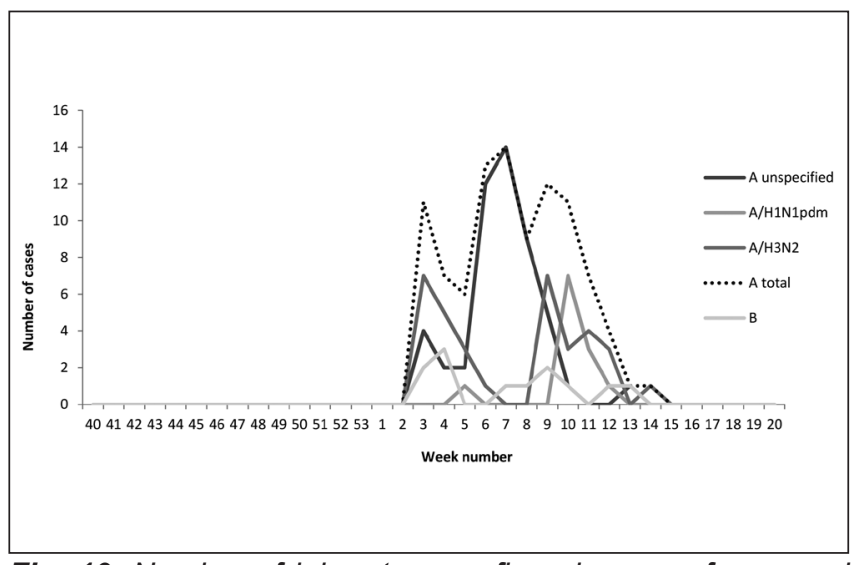

Fig. 10. Number of laboratory-confirmed cases of seasonal influenza (all types) per week, 2013/2014, $n=108$.

was reported at week 11 (middle of March), while the last detection of influenza B virus was at week 14 (first week of April), keeping in mind that detection of influenza $B$ started later than influenza $\mathrm{A}$, at week 3 (middle of January).

During the 2013/2014 season, 108 samples were positive for influenza out of 206 samples tested. Of them 96 (88.88\%) were positive for influenza $A$, and $12(11.11 \%)$ positive for influenza B (Figure 10). Subtype A/H3N2 was detected among 34 (35.42\%) versus $12(12.5 \%)$ of influenza A/H1N1pdm positive. The season started at week 3 (middle of January), with the peak at week 7 (middle of February), and stopped at week 13 (last week of March).

In three seasons (2010/2011, 2012/2013 and 2013/2014) incidence of laboratory confirmed cases was highest among children aged $0-4$ years, while in the 2011/2012 season the incidence was highest among persons more than 65 years old (Tables 2, 3, 4 and 5). Data from the epidemiological surveillance suggest that incidence is inversely related to age, and for all seasons during the 2010-2014 period, the highest incidence was observed in the age group $0-4$, while the lowest was observed in the age group $65+$ years (Table 6). During laboratory surveillance throughout the observed period the highest incidence $(3,208.5 / 100,000)$ of ILI cases was noted in the 2010/2011 season among the age group 0-4 years.

\section{DISCUSSION}

Influenza epidemics of different magnitudes occur every winter season. To be able to respond and control influenza re-

Table 2. Number of lab confirmed cases and incidence per 100,000 in 2010/2011 season

\begin{tabular}{|c|c|c|c|c|c|c|c|c|c|c|c|c|}
\hline \multirow{2}{*}{$\begin{array}{l}\text { Age } \\
\text { groups }\end{array}$} & \multicolumn{6}{|c|}{ Number of lab confirmed cases } & \multicolumn{6}{|c|}{ Incidence per 100,000} \\
\hline & $\begin{array}{c}\text { A unsub- } \\
\text { typed }\end{array}$ & $\begin{array}{c}\mathrm{A} / \mathrm{H} 1 \mathrm{~N} 1 \\
\mathrm{pdm}\end{array}$ & $\mathrm{A} / \mathrm{H} 3 \mathrm{~N} 2$ & A Total & B & Total & $\begin{array}{c}\text { A unsub- } \\
\text { typed }\end{array}$ & $\begin{array}{c}\text { A/H1N1 } \\
\text { pdm }\end{array}$ & $\mathrm{A} / \mathrm{H} 3 \mathrm{~N} 2$ & A Total & B & Total \\
\hline $0-4$ & 0 & 6 & 2 & 8 & 10 & 18 & 0.0 & 5.3 & 1.8 & 7.0 & 8.8 & 15.8 \\
\hline $5-14$ & 0 & 3 & 1 & 4 & 12 & 16 & 0.0 & 1.2 & 0.4 & 1.6 & 4.9 & 6.5 \\
\hline $15-64$ & 1 & 23 & 2 & 26 & 14 & 40 & 0.1 & 1.6 & 0.1 & 1.8 & 1.0 & 2.8 \\
\hline $65+$ & 1 & 0 & 1 & 2 & 1 & 3 & 0.4 & 0.0 & 0.4 & 0.8 & 0.4 & 1.3 \\
\hline Total & 2 & 32 & 6 & 40 & 37 & 77 & 0.1 & 1.6 & 0.3 & 1.9 & 1.8 & 3.7 \\
\hline
\end{tabular}


Table 3. Number of lab confirmed cases and incidence per 100,000 in 2011/2012 season

\begin{tabular}{|l|c|c|c|c|c|c|}
\hline \multirow{2}{*}{ Age groups } & \multicolumn{3}{|c|}{ Number of lab confirmed cases } & \multicolumn{3}{c|}{ Incidence per 100,000 } \\
\cline { 2 - 8 } & A unsubtyped & A/H3N2 & A Total & A unsubtyped & A/H3N2 & A Total \\
\hline 0-4 years & 1 & 1 & 2 & 0.9 & 0.9 & 1.8 \\
\hline $5-14$ years & 0 & 6 & 6 & 0.0 & 2.4 & 2.4 \\
\hline 15-64 years & 6 & 28 & 34 & 0.4 & 1.9 & 2.3 \\
\hline $65+$ years & 1 & 8 & 9 & 0.4 & 3.3 & 3.8 \\
\hline Total & 8 & 43 & 51 & 0.4 & 2.1 & 2.5 \\
\hline
\end{tabular}

Table 4. Number of lab confirmed cases and incidence per 100,000 in 2012/2013 season

\begin{tabular}{|c|c|c|c|c|c|c|c|c|c|c|c|c|}
\hline \multirow{2}{*}{$\begin{array}{l}\text { Age } \\
\text { groups }\end{array}$} & \multicolumn{6}{|c|}{ Number of lab confirmed cases } & \multicolumn{6}{|c|}{ Incidence per 100,000} \\
\hline & $\begin{array}{c}\text { A unsub- } \\
\text { typed }\end{array}$ & $\begin{array}{c}\mathrm{A} / \mathrm{H} 1 \mathrm{~N} 1 \\
\mathrm{pdm}\end{array}$ & $\mathrm{A} / \mathrm{H} 3 \mathrm{~N} 2$ & A Total & B & Total & A & $\begin{array}{c}\mathrm{A} / \mathrm{H} 1 \mathrm{~N} 1 \\
\mathrm{pdm}\end{array}$ & $\mathrm{A} / \mathrm{H} 3 \mathrm{~N} 2$ & A Total & B & Total \\
\hline $0-4$ & 1 & 9 & 3 & 13 & 3 & 16 & 0.9 & 7.9 & 2.6 & 11.4 & 2.6 & 14.1 \\
\hline $5-14$ & 0 & 12 & 4 & 16 & 1 & 33 & 0.0 & 4.9 & 1.6 & 6.5 & 0.4 & 13.3 \\
\hline $15-64$ & 4 & 37 & 4 & 45 & 4 & 94 & 0.3 & 2.5 & 0.3 & 3.1 & 0.3 & 6.5 \\
\hline $65+$ & 2 & 4 & 3 & 9 & 1 & 19 & 0.8 & 1.7 & 1.3 & 3.8 & 0.4 & 7.9 \\
\hline Total & 7 & 62 & 14 & 83 & 9 & 92 & 0.3 & 3.0 & 0.7 & 4.0 & 0.4 & 4.5 \\
\hline
\end{tabular}

Table 5. Number of lab confirmed cases and incidence per 100,000 in 2013/2014 season

\begin{tabular}{|c|c|c|c|c|c|c|c|c|c|c|c|c|}
\hline \multirow{2}{*}{$\begin{array}{l}\text { Age } \\
\text { groups }\end{array}$} & \multicolumn{6}{|c|}{ Number of lab confirmed cases } & \multicolumn{6}{|c|}{ Incidence per 100,000} \\
\hline & $\begin{array}{c}A \text { unsub- } \\
\text { typed }\end{array}$ & $\begin{array}{c}\mathrm{A} / \mathrm{H} 1 \mathrm{~N} 1 \\
\mathrm{pdm}\end{array}$ & $\mathrm{A} / \mathrm{H} 3 \mathrm{~N} 2$ & A Total & B & Total & A & $\begin{array}{c}\mathrm{A} / \mathrm{H} 1 \mathrm{~N} 1 \\
\mathrm{pdm}\end{array}$ & $\mathrm{A} / \mathrm{H} 3 \mathrm{~N} 2$ & A Total & B & Total \\
\hline $0-4$ & 6 & 3 & 5 & 14 & 3 & 17 & 5.3 & 2.6 & 4.4 & 12.3 & 2.6 & 14.9 \\
\hline $5-14$ & 5 & 2 & 6 & 13 & 1 & 14 & 2.0 & 0.8 & 2.4 & 5.3 & 0.4 & 5.7 \\
\hline $15-64$ & 22 & 2 & 15 & 39 & 6 & 45 & 1.5 & 0.1 & 1.0 & 2.7 & 0.4 & 3.1 \\
\hline $65+$ & 13 & 4 & 6 & 23 & 1 & 24 & 5.4 & 1.7 & 2.5 & 9.6 & 0.4 & 10.0 \\
\hline Total & 46 & 11 & 32 & 89 & 11 & 100 & 2.2 & 0.5 & 1.6 & 4.3 & 0.5 & 4.9 \\
\hline
\end{tabular}

Table 6. Seasonal ILI incidence per age groups, 2010-2014 season

\begin{tabular}{|l|c|c|c|c|}
\hline \multirow{2}{*}{ Age groups } & \multicolumn{4}{|c|}{ Seasonal incidence ILI cases per 100,000 } \\
\cline { 2 - 5 } & $\mathbf{2 0 1 0 / 2 0 1 1}$ & $\mathbf{2 0 1 1 / 2 0 1 2}$ & $\mathbf{2 0 1 2 / 2 0 1 3}$ & $\mathbf{2 0 1 3 / 2 0 1 4}$ \\
\hline 0-4 years & $3,208.5$ & $1,276.4$ & $2,139.4$ & $2,399.3$ \\
\hline $5-14$ years & $2,253.2$ & 811.1 & $1,958.6$ & $1,766.2$ \\
\hline 15-64 years & $1,183.0$ & 386.4 & $1,067.7$ & $1,303.8$ \\
\hline $65+$ years & 619.3 & 276.9 & 942.7 & $1,258.2$ \\
\hline
\end{tabular}

lated threats, efficient surveillance is the basic step. Virological and epidemiological surveillance must occur simultaneously to maximize surveillance efforts.

Until the pandemic season of 2009, there was limited data regarding types and subtypes of circulating influenza viruses in the country. With the introduction of real-time RT-PCR detection of influenza viruses for the first time in 2009 in Macedonia, continuous and reliable lab surveillance of influenza was established, resulting in increased likelihood of positive diagnosis, and making sampling more valuable for the clinicians, epidemiologists and health authorities.
For all four described seasons after the 2009/2010 pandemic, the cumulative number of positive samples was nearly three times less compared to the number of confirmed H1N1pdm samples during the 2009 pandemic (8). Nonetheless, a higher number of samples are tested using real-time PCR and therefore a higher number of cases are verified in comparison with seasons before the pandemic. The possible reasons for a lower percentage of positive samples in the first two seasons $2010 / 2011(\mathrm{RD}=-18.7 \%$; $\mathrm{RR}=0.65)$ and $2011 / 2012(\mathrm{RD}=-16.1 \% ; \mathrm{RR}=0.70)$ after the pandemic is the result of higher awareness of doctors about influenza and the risk of possible complications leading to higher 
sampling of patients with no clear presentation of case definition for ILI. Other possible contributing factors may be sampling time greater than 5 days, sampling after antiviral treatment, sampling after failure of antibiotic treatment or during a mild-season such as 2011/2012 defined by a small number of outpatient visits and a small number of severe acute respiratory illness (SARI) cases (9). The possible reason for a higher percentage of positive samples in the second two seasons could be that clinicians were more familiar with the case definition.

It is obvious that all post-pandemic seasons started later than the pandemic season, usually at the beginning of the year, and ended in week 14 (end of April - first week of May), whilst the second wave of 2009 pandemic started in week 44 (October). The start of the first two post-pandemic influenza seasons was delayed compared to the second two post-pandemic influenza seasons. In all four seasons influenza A types (83.25\%) were predominant over B types (16.75\%). Additionally, subtype A/ H1N1pdm (45.27\%) was predominant over A/H3N2 (31.06\%), alike in the WHO European region (10-13).

The influenza season 2010/2011 started at the end of December, with influenza $\mathrm{B}$ virus as it was expected having in mind the development of immunity against influenza A/H1N1pdm as result of the incidence of disease, vaccination during the pandemic season and decreased immunity for influenza B. The duration of the influenza B season was two months - until the first part of February with a peak at the end of January. Influenza A virus emerged at the end of January with a peak in the middle of March. After two months of circulation, the last detected influenza A/ H1N1pdm was reported at the beginning of April. Total duration of the influenza season was three months. Influenza seasons most often peak in January or February (14). In other countries, a greater proportion of influenza B was seen early in the season and as the season progressed, an increasing proportion of influenza $\mathrm{A} / \mathrm{H} 1 \mathrm{~N} 1 \mathrm{pdm}$ viruses were found (14). Influenza activity started in week 52 of 2010 in several countries in Europe and showed evidence of West to East spread. The WHO described the season as relatively mild compared to the $2009 / 2010$ season with peak activity at weeks 2 to 3 . Unusually, influenza B started before influenza $\mathrm{A}$, and there was a period of co-circulation of both viruses compared with previous seasons where the circulation of influenza B occurred after influenza A circulation had declined. Overall, type A viruses predominated. Between countries there were considerable variations in the proportions of type A and type $\mathrm{B}$ circulation with A being predominant. In a number of Eastern countries of the WHO region influenza B dominated at the start of the season but was overtaken by A/H1N1pdm viruses (10). Influenza activity peaked earliest in some West European countries like Spain at week 2, in Russian federation and Moldova at week 5, in Slovenia at weeks 2 to 5, and Croatia at week 7 (15, 16). As with every influenza season, the proportion of different virus types and subtypes varied by geographic region and week. Influenza A/H1N1pdm and influenza B co-circulated in the European region (15). Of the positive specimens, $74 \%$ were influenza A viruses, and $26 \%$ were influenza B viruses. Among the influenza A viruses, $62 \%$ were influenza $\mathrm{A} / \mathrm{H} 3 \mathrm{~N} 2$ and $38 \%$ were influenza $\mathrm{A} / \mathrm{H} 1 \mathrm{~N} 1 \mathrm{pdm}$ (17). The season started to subside at week 9 and lasted in April $(14,15)$. There was influenza A domination in Spain, England, Germany, Moldova, Greece, Russian federation, Israel, and Macedonia (18).
The 2011/2012 season started one week before the previous season with a peak in the middle of March. The season was longer than that of the following two seasons. The season began late and was mild. During the 2011/2012 season, ILI remained low throughout February and did not peak until mid-March (19). After four months of circulation, detection of influenza A/H3N2 stopped at the end of April. During this particular season, there was no detection of influenza A/H1N1pdm and influenza B as result of developed immunity in the population and a lack of antigenic shift evidence which can cause the introduction of a new virus. Across the whole WHO European region, influenza activity increased slowly over consecutive weeks since December and increased in January and February before peaking in mid-March (weeks 10 to 12 ) with type A virus (94.6\%) (11). Influenza A/H3N2 viruses predominated $(95.8 \%)$ overall, but influenza A/H1N1pdm and influenza B viruses also circulated widely $(9,20)$.

The 2012/2013 influenza season was three months long. Other countries in Europe, where the season started earlier than the 2011/2012 influenza season, influenza positivity began increasing slowly from week 46 of 2012. The influenza season in Macedonia started at the beginning of January with influenza A/H3N2 followed by dominant influenza A/H1N1pdm immediately after the start of the circulation. The peak of activities between European countries and Macedonia was similar at week 5 and 4, respectively. Across the whole WHO European Region, type A viruses (70\%) predominated over type B (30\%), but these relative proportions were slightly different for Western (EU/EAA) countries $(68 \%$ and $32 \%$ ) and Eastern countries (82\% and $18 \%)$. West to East spread across the region is clear. Macedonia is close to Eastern countries with $84.95 \%$ for influenza A and $15.4 \%$ for influenza B. We observe lower co-circulation of influenza B later than influenza $\mathrm{A}$, which is often the pattern of influenza season. Within type A, the H1N1pdm subtype (72.9\% in Macedonia versus $71 \%$ in $\mathrm{WHO}$ region) predominated over the $\mathrm{H} 3$ subtype $(12.3 \%$ in Macedonia versus 29\% in WHO region). This represents a reversal of the situation in 2011/2012. Overall, influenza activity across the region was greater than that in the 2011/2012 season (12).

The 2013/2014 influenza season in Macedonia and in the WHO region started later than previous seasons, with high domination of influenza A $(88,88 \% / 95)$ as opposed to influenza B. Among influenza A subtypes in Macedonia, influenza A/H3N2 dominated over influenza A/H1N1pdm against slightly more prevalent (59\%) $\mathrm{A} / \mathrm{H} 1 \mathrm{~N} 1 \mathrm{pdm}$ subtype compared to $\mathrm{A} / \mathrm{H} 3 \mathrm{~N} 2$ viruses $(41 \%)$ in other WHO countries. But there are some variations in different countries like Slovenia and Turkey with domination of influenza A over B and H3 over H1 (13, 20, 21). The peak of influenza activity in Macedonia was similar to other European countries such as Austria with peak from weeks 7 to 12 (22)

The effect on the society depends on the characteristics of circulating virus and population immunity in different age groups. Also, certain age groups may be hit harder by one type of influenza virus than another (e.g. A/H1N1pdm virus has disproportionately affected people younger than 65 years of age than those older than 65 whereas $\mathrm{A} / \mathrm{H} 3 \mathrm{~N} 2$ virus often affects those older than 65 years $(8,19)$. All influenza seasons had big health effects on every age group. In seasons with domination of influenza A/H1N1pdm, we observed higher detection among persons aged $0-14$ years, and in the season with domination of A/H3N2 the most affected were persons aged more than 65 years. 


\section{CONCLUSIONS}

The timing of influenza can be unpredictable and can vary from season to season. Our results revealed that the past four post-pandemic influenza seasons in the Republic of Macedonia has been virologically very different.

Seasonal influenza activity used to begin as early as December and continue to the end of April. Influenza activity in Macedonia most commonly peaks in the middle of January or February. Based on the data for all four seasons of influenza in Macedonia as well as in the WHO region influenza activity had mixed aetiology, with predomination of influenza A and A/H1N1pdm. There was only one season, 2011/2012, demonstrating circulation of only influenza A/H3N2 viruses. Influenza B viruses usually occur later than influenza A viruses, with the exception of the first post-pandemic season. As result of the introduction of realtime RT-PCR test for influenza, there is increased likelihood of positive diagnosis, and sampling has become more valuable for the clinicians, epidemiologists and health authorities. The most affected patients were aged $0-4$ and 5-14 years. Only in case of influenza A/H3N2, the most affected persons were older than 64 years. The data for the burden of disease at different age and risk groups are important for developing control policies by health authorities. According to both epidemiological and laboratory data, the highest incidence was observed among children up to 4 years of age. This is a matter of concern since influenza vaccination is lowest among these children.

The pattern of influenza seasons in Macedonia is similar to some countries in the WHO region, though with some unique characteristics. Future directions include implementation of a sentinel surveillance system and having a complete view of the circulation of influenza viruses, their variants or novel strains.

\section{Collaborators}

E. Dokic Trajkovska ${ }^{3}$, Z. Milenkovic ${ }^{2}$, K. Stavridis ${ }^{1}$, E. Janceska ${ }^{1}$, G. Kuzmanovska' ${ }^{1}$ Shaban Memeti ${ }^{1}$

\section{Acknowledgements}

Our thanks to doctors Jovanka Kostovska and Suzana Manevska from the Sector for Preventive Health Care at the Ministry of Health, for their support rendered to the surveillance system. We extend our gratitude to all Lab and Epi staff at the Institute of Public Health, doctors at the Clinic for Infectious Disease in Skopje and at the 10 regional Centres of Public Health in Macedonia. Special thanks to the CDC influenza division and reagent resources for providing CDC real-time RT-PCR reagents for lab surveillance of influenza.

\section{Conflict of Interests}

None declared

\section{REFERENCES}

1. Stöhr K. The global agenda on influenza surveillance and control. Vaccine. 2003 May 1;21(16):1744-8.

2. World Health Organization. International Health Regulations 2005 [Internet]. Geneva: WHO; 2008 [cited 2017 Feb 8]. Available from: http:// www.who.int/ihr/9789241596664/en/index.html.

3. World Health Organization. WHO Regional Office for Europe Guidance for influenza surveillance in humans. Copenhagen: WHO Regional Office for Europe; 2009.
4. Law on protection of the population against communicable diseases. Official gazette of Republic of Macedonia. 2004;(66). (In Macedonian.)

5. Rulebook for reporting communicable diseases. Official gazette of Republic of Macedonia. 2009;(46). (In Macedonian.)

6. Rulebook for criteria used for case definition of communicable diseases. Official gazette of Republic of Macedonia. 2011;(10). (In Macedonian.)

7. CDC Protocol of realtime RTPCR for swine influenza A (H1N1) [Internet]. Atlanta: WHO Collaborating Centre for influenza at CDC; 2009 [cited 2015 Jan 1]. Available from: http://www.who.int/csr/resources/ publications/swineflu/CDCrealtimeRTPCRprotocol 20090428.pdf.

8. Bosevska G, Janceska E, Memeti S, Kuzmanovska G, Mikik V, Kondova Topuzovska I, et al. Laboratory surveillance during pandemic influenza season 2009/2010 in Republic of Macedonia. Pril (Makedon Akad Nauk Umet Odd Med Nauki). 2013;34(2):51-8.

9. Centers for Disease Control and Prevention (CDC). Update: influenza activity - United States, 2011-12 season and composition of the 201213 influenza vaccine. MMWR Morb Mortal Wkly Rep. 2012 Jun $8 ; 61(22): 414-20$.

10. National Institute for Medical Research, WHO Influenza Centre London. Report prepared for the WHO annual consultation on the composition of influenza vaccine for the Northern Hemisphere, 14th - 17 th February 2011. London: NIMR; 2011.

11. National Institute for Medical Research, WHO Influenza Centre London. Report prepared for the WHO annual consultation on the composition of influenza vaccine for the Northern Hemisphere, 20th - 22nd February 2012. London: NIMR; 2012.

12. National Institute for Medical Research, WHO Influenza Centre London. Report prepared for the WHO annual consultation on the composition of influenza vaccine for the Northern Hemisphere 2013/14, 18 th - 20th February 2013. London: NIMR; 2013.

13. National Institute for Medical Research, WHO Influenza Centre London. Report prepared for the WHO annual consultation on the composition of influenza vaccine for the Northern Hemisphere 2014/15, 17th - 19th February 2014. London: NIMR; 2014.

14. Centers for Disease Control and Prevention. Influenza (Flu). 2010-2011 Influenza (Flu) Season [Internet]. Atlanta CDC; 2011 [cited 2017 Feb 8]. Available from: http://www.cdc.gov/flu/pastseasons/1011season.htm.

15. Plata F. Regional overview 2010/2011 Primary care surveillance. In: Joint ECDC/WHO Regional Office for Europe Meeting on Influenza Surveillance; 2011 Jun 7-9; Ljubljana, Slovenia.

16. Prosenc K, Sočan M. Epidemiological and virological surveillance of influenza-like illness in Slovenia. In: Joint ECDC/WHO Regional Office for Europe Meeting on Influenza Surveillance; 2011 Jun 7-9; Ljubljana, Slovenia.

17. Centers for Disease Control and Prevention (CDC). Update: influenza activity - United States, 2010-11 season, and composition of the 2011-12 influenza vaccine. MMWR Morb Mortal Wkly Rep. 2011 Jun 3;60(21):705-12.

18. Global Virological Surveillance (Focus on Europe): 2010/11 Influenza Season. In: Joint ECDC/WHO Regional Office for Europe Meeting on Influenza Surveillance; 2011 Jun 7-9; Ljubljana, Slovenia.

19. Centers for Disease Control and Prevention. Influenza (Flu). 2011-2012 Influenza Season [Internet]. Atlanta CDC; 2012 [cited 2015 Jan 4]. Available from: https://www.cdc.gov/flu/pastseasons/1112season.htm.

20. Gross D. Season report 2013-14. In: Fourth Joint WHO Regional Office for Europe/ECDC Meeting on Influenza Surveillance; 2014 Jun 11-13; Vienna, Austria.

21. Centers for Disease Control and Prevention. Influenza (Flu). 2013-2014 Influenza Season [Internet]. Atlanta CDC; 2014 [cited 2015 Jan 4]. Available from: http://www.cdc.gov/flu/pastseasons/1314season.htm.

22. Popow-Kraupp T. Influenza surveillance in Austria. In: Fourth Joint WHO Regional Office for Europe/ECDC Meeting on Influenza Surveillance; 2014 Jun 11-13; Vienna, Austria. 